OPEN ACCESS

Edited by:

Yan Mark Yufik,

Virtual Structures Research Inc.

United States

Reviewed by:

Derek L. Buhl,

Takeda, United States

Laura M. Harrison,

Tulane University, United States

*Correspondence:

Bang-Tao Chen

medisci@163.com

tThese authors have contributed equally to this work

Received: 01 May 2021 Accepted: 19 August 2021 Published: 29 September 2021

Citation:

Wang C, Zhang T, He L, Fu J-Y, Deng $H-X, X u e X-L$ and Chen B-T

(2021) Bacterial Translocation

Associates With Aggression in

Schizophrenia Inpatients.

Front. Syst. Neurosci. 15:704069. doi: 10.3389/fnsys.2021.704069

\section{Bacterial Translocation Associates With Aggression in Schizophrenia Inpatients}

\author{
Chong Wang ${ }^{1+}$, Teng Zhang ${ }^{1 \dagger}$, Lei He ${ }^{1}$, Ji-Yong Fu ${ }^{1}$, Hong-Xin Deng ${ }^{1}$, Xiao-Ling Xue ${ }^{2}$ and \\ Bang-Tao Chen ${ }^{3 *}$ \\ 1 Department of Psychiatry, Zhumadian Psychiatric Hospital (The Second People's Hospital of Zhumadian), Zhumadian, \\ China, ${ }^{2}$ Department of Hematology, The Third Affiliated Hospital of Chongqing Medical University, Chongqing, China, \\ ${ }^{3}$ Department of Dermatology, Chongqing University Three Gorges Hospital, Chongqing, China
}

Objective: Accumulating evidence indicates that inflammation abnormalities may contribute to aggression behaviors in psychotic patients, however, the possible sources of inflammation remain elusive. We aimed to evaluate the associations among aggression, inflammation, and bacterial translocation (BT) in aggression-affected schizophrenia (ScZ) inpatients with 2 weeks of antipsychotics discontinuation.

Methods: Serum specimens collected from 112 aggression and 112 non-aggression individuals with ScZ and 56 healthy adults were used for quantifications of inflammation- or BT-related biomarkers. Aggression severity was assessed by Modified Overt Aggression Scale (MOAS).

Results: Proinflammation phenotype dominated and leaky gut-induced BT occurred only in cases with ScZ with a history of aggression, and the MOAS score positively related to levels of $\mathrm{C}$-reactive protein, interleukin (IL)-6, IL-1 $\beta$, and tumor necrosis factor- $\alpha$. Furthermore, serum levels of BT-derived lipopolysaccharide (LPS), as well as LPS-responded soluble CD14, were not only positively correlated with levels of above proinflammation mediators but also the total MOAS score and subscore for aggression against objects or others.

Conclusion: Our results collectively demonstrate the presence of leaky gut and further correlate BT-derived LPS and soluble CD14 to onset or severity of aggression possibly by driving proinflammation response in inpatients with ScZ, which indicates that BT may be a novel anti-inflammation therapeutic target for aggression prophylaxis.

Keywords: schizophrenia, aggression, bacterial translocation, inflammation, association

\section{INTRODUCTION}

Schizophrenia (ScZ) is a chronic and heterogeneous psychiatric syndrome characterized by recurrent episodes of acute psychosis alternating with periods of full or partial remission. Globally, ScZ affects $\sim 1 \%$ of the population and occurs mainly in individuals in the late adolescence or early adulthood (Kahn et al., 2015; Charlson et al., 2018). It covers a broad spectrum of clinical symptoms including positive symptoms (delusions, hallucinations, etc.), negative symptoms (anhedonia, social withdrawal, poverty of thought, etc.), and cognitive dysfunction. Current treatment modalities are available only for symptoms mitigation, thus, significant disability, 
insupportable psychosocial burdens, and premature mortality are of great concerns (Tiihonen et al., 2017; Stepnicki et al., 2018).

Compared with the general population, inpatients with ScZ are four to seven times more likely to commit aggression acts involving verbal threat, assault, and homicide, which brings a great challenge for both mental health services and public safety (Cho et al., 2019). Aggression is more inclined to be an independent entity. The manifold pathogenesis of aggression in ScZ is complicated by elevated serum C-reactive protein (CRP) and increased ratio of CRP to interleukin (IL)-10, which arouses increasing concerns about the role of systemic inflammation in the onset or severity of aggression in ScZ (Barzilay et al., 2016; Das et al., 2016; Zhang et al., 2017). Inflammation phenotype involves the integration of various pro-/anti-inflammatory cytokines. Interleukin-6, IL-1 $\beta$, and tumor necrosis factor (TNF)$\alpha$ are well-proved proinflammatory cytokines responsible for initiation and exacerbation of inflammation, and the serum levels of them were demonstrated to be significantly upregulated in patients with ScZ in most of the related studies (Lesh et al., 2018; Momtazmanesh et al., 2019). Although other cytokines such as interferon (IFN)- $\gamma$, IL-4, IL-17, IL-10, and transforming growth factor (TGF)- $\beta$ were also proved to be linked with ScZ, they may promote or suppress inflammation response in the different subsets of cases with ScZ (Lesh et al., 2018; Momtazmanesh et al., 2019). Crossing blood-brain barrier, the peripheral cytokines precipitate changes in mood and behavior through hypothalamic-pituitary-adrenal axis (Petra et al., 2015; Singh et al., 2019; Misiak et al., 2020), which lays a structural foundation for studying the involvement of inflammation in aggression. However, the mentioned cytokines except serum CRP are rarely profiled and potential sources of peripheral inflammation, with exception of being overweight or lack of dental care, are seldom explored (Fond et al., 2021) in individuals with aggression (Ag)-affected ScZ (ScZ-Ag).

Interestingly, inflammation abnormalities could be caused by alterations in the gut microbiome and the recent evidence from human metabolomics suggested a correlation between enteric dysbacteriosis and dysfunction of neurochemical pathways including inflammation activation underlying aggression in patients with ScZ (Severance et al., 2016; Manchia and Fanos, 2017; Chen et al., 2021; Zeng et al., 2021). Changes in gut microbiota may compromise the integrity of the intestinal tract (leaky gut) and subsequently cause a higher translocation rate of bacterial immunogenic components such as bacterial DNA (BactDNA) and lipopolysaccharide (LPS) from gut into peripheral circulation, which in turn activate immunoinflammatory signaling (Francés et al., 2007; Martin-Subero et al., 2016). The so-called bacterial translocation (BT) was extensively proved to be correlated with various inflammationinvolved diseases and with negative symptoms or neurocognitive impairments in deficit cases with ScZ (Caso et al., 2016; Maes et al., 2019a; Severance et al., 2020). However, the occurrence of leaky gut-related BT and its association with systemic inflammation in ScZ-Ag are poorly investigated.

Taken together, we hypothesize that proinflammation cytokines characterize the aggression behaviors in patients with $\mathrm{ScZ}$ and increased intestinal permeability-caused BT is one of the main culprits for the tuning process of inflammation. With regard to this, we determined serum levels of aforementioned inflammation cytokines, leaky gut and BT-related biomarkers, and further assessed the correlations between BT biomarkers and inflammation cytokines or the severity of aggression, in the hope of providing more convincing evidence for BT-derived inflammatory pathogenesis of aggression in ScZ.

\section{MATERIALS AND METHODS}

\section{Study Population}

The prospective and controlled investigation was conducted in inpatients with ScZ with or without aggression behaviors within 1 week prior to admission during November 2019 to November 2020 in the Second People's Hospital of Zhumadian, a tertiary psychiatric hospital in Henan Province, China. At sample collection, all included inpatients with ScZ were at least 2 weeks of antipsychotics discontinuation. Inpatients with ScZ with the presence of aggression behaviors within 1 week prior to admission and absence of any aggression behaviors during disease course before enrollment were classified into ScZ-Ag and NScZ-Ag groups, respectively.

For comparison, age-, gender-, and body mass index (BMI)matched healthy volunteers recruited during the same period with no history of psychiatric or medical illness were set as control (Ctrl group) and the ratio of healthy volunteers: cases with ScZ-Ag is 1:2. All the subjects were aged $\geq 18$ years. The diagnosis was made by two board-certified psychiatrists according to the 10th edition of the international classification of diseases (ICD-10) criteria for ScZ. Exclusion criteria included: (a) aggression behaviors not within 1 week prior to admission; (b) pregnant or lactating women; (s) presence of any other psychoses including affective disorder or substance abuse; (d) comorbidity with severe somatic diseases or neurological diseases; (e) comorbidity with other medical conditions such as parenchymal organ-specific diseases, immune-related diseases, hematological diseases, gastrointestinal diseases, and any history of gastrointestinal surgeries; (f) use of systemic corticosteroids, any other immunosuppressive therapy, and oral probiotics in the recent 3 months; $(\mathrm{g})$ inpatients with fever $\left(>37.9^{\circ} \mathrm{C}\right)$ or those who were treated with antibiotics, antipyretics or antiinflammatory medications in the recent 2 weeks. The study was approved by and carried out under the guidelines of the Ethics Committee of the Hospital, and written informed consent was obtained from all the healthy volunteers, the inpatients or the guardians of inpatients (if the patients were unable to sign consent because of poor intelligence) at the time of recruitment.

\section{Subjects Profiles}

A structured questionnaire was used to collect data on general sociodemographic variables (age, gender, occupation, education background, ethnicity, height and weight, family income, living circumstance and marriage status), health status (medical history, current medications and family history), and living habits (alcohol intake and smoking pattern) in all the participants. In inpatients with $\mathrm{ScZ}$, the information on specific conditions 
including the onset of illness and the type of aggression was inquired.

\section{Clinical Assessments}

Modified Overt Aggression Scale (MOAS) was used to characterize aggression behaviors observed within the past 1 week. It involves four subscales and a score from zero to four is assigned for each type of aggression with zero indicating no aggression and higher scores pointing to increasing severity. The score of each subscale is then multiplied by a predefined loading (one for verbal aggression, two for aggression against objects, three for self-aggression, and four for aggression against other people) and the sum of each subscale-weighted score (range $0-40$ ) is referred to the total score. Inpatient with a total score of zero or only having a score of one or more for verbal aggression was classified as being the non-aggressive (Huang et al., 2009). The presence and severity of each psychiatric symptom in cases with ScZ were evaluated by the positive and negative syndrome scale (PANSS) involving positive symptom subscale (seven items), negative symptom subscale (seven items), general psychopathological subscale (16 items), and supplemental items (three items). Each item on the subscale score from one to seven base on the frequency and severity of the symptom (Kelley et al., 2013).

\section{Blood Sampling and Laboratory Detection}

Fasting peripheral blood samples were collected from all the subjects at 8:00 a.m. Blood cell count and liver function were examined routinely. The protein levels of indicators assessed by enzyme-linked immunosorbent assay (ELISA) in this study involved CRP (\#E007462, 3ABio, Shanghai, China), IL-6 (\#E000482, 3ABio, Shanghai, China), IL-1 $\beta$ (\#E001772, 3ABio, Shanghai, China), IL-4 (\#DG10308H, Dogesce, Beijing, China), IL-10 (\#DG10495H, Dogesce, Beijing, China), IL17 (\#DG10431H, Dogesce, Beijing, China), IFN- $\gamma$ (\#C608-01, GenStar, Beijing, China), TNF- $\alpha$ (\#489204, Cayman, Michigan, USA), TGF- $\beta$ (\#DG10113H, Dogesce, Beijing, China); leaky gut-related biomarkers[intestinal fatty acid-binding protein (I-FABP, \#DFBP20, R\&D Systems, Minnesota, USA) and Claudin-3 (\#abx250611, Abbexa, Cambridge, UK)]; BT-related biomarkers[LPS (\#DG11072H, Dogesce, Beijing, China), soluble CD14 (sCD14, \#DC140, R\&D Systems, Minnesota, USA), and endotoxin core antibody (EndoCAb, \#E013362, 3ABio, Shanghai, China)]. Assays were performed according to the specifications of the manufacturer and the detection limits were in line with the instructions of the manufacturer. Each serum sample was measured in duplicate. All the plates were read by the I Mark ${ }^{\mathrm{TM}}$ Micro plate Reader (Bio-Rad, Hercules, California, United States).

\section{Quantification of BactDNA Fragments}

Quantification of circulating BactDNA fragments and quality control were performed as described previously (Such et al., 2002; Ericsen et al., 2016). To avoid potentially bacterial contamination of molecular biology reagents, all the specimens were processed in airflow chambers by the same investigator and all the tubes were never exposed to free air. To remove potentially confounding $16 \mathrm{~S}$ rDNA contamination, six tubes of prepared diethyl pyrocarbonate (DEPC) water were set as negative controls and the processes of water from DNA extraction to quantitative PCR (qPCR) were completely synchronized with those of blood.

Genomic DNA was extracted from $200 \mu \mathrm{l}$ of serum or DEPC water using QIAmp DNA Blood Minikit (Qiagen, Hilden, Germany) according to the instructions of the manufacturer and DNA was eluted in a $100 \mu \mathrm{l}$ final volume. BactDNA levels were determined by qPCR in an amplification reaction of 20 $\mu l$ with forward primer (5'-AGAGGGTGATCGGCCACA-3') and reverse primer ( $5^{\prime}$-TGCTGCCTCCCGTAGGAGT- $\left.3^{\prime}\right)$, the universal eubacterial primers of a conserved region of $16 \mathrm{~S}$ rDNA gene (Francés et al., 2004). The amplification conditions for the 59 base pairs of DNA fragments were $95^{\circ} \mathrm{C}$ for $10 \mathrm{~min}$, followed by 45 cycles at $95^{\circ} \mathrm{C}$ for $15 \mathrm{~s}$ and $60^{\circ} \mathrm{C}$ for $60 \mathrm{~s}$. Each sample was amplified in triplicate and the BactDNA content was calculated according to a standard curve that generated from serial dilutions of plasmid DNA containing known copy numbers of the template. The final circulating BactDNA concentration was calculated by subtracting the proportion of $16 \mathrm{~S}$ rDNA copies $/ \mu \mathrm{l}$ detected in water controls from those in blood.

\section{Statistical Analyses}

Statistical analysis of the data compiled in Excel databank was conducted using SPSS/PC software (Version 19.0 for Windows; SPSS Inc., China). Categorical and continuous variables were expressed as number (\%) or mean (M) $\pm \mathrm{SD}$, respectively. Normal distribution of raw data was inspected by Kolmogorov-Smirnov tests, and IL-17, IGF- $\beta$, and EndoCAb were logarithmically transformed to achieve Gaussian distributions. There were no outliers in MOAS score, PANSS score, cytokines, and bacterial measures by inspection of related boxplots. For comparison of demographic information and clinical characteristics at baseline among groups, Fisher's exact Chi-square test or one-way ANOVA were conducted except specification. Analysis of covariance (ANCOVA) controlling for age, gender, BMI, and course with ScZ was used to analyze cytokines and bacterial measures among the three groups, and Bonferroni's multiple comparison test that can calculate the corrected statistical significance for multiple comparisons was performed for post-hoc analysis of pairwise comparisons. Partial correlation analysis controlling for episodes with ScZ, course with ScZ, income levels, marriage status, education background, and occupation was used to determine the relationship between clinical symptoms and inflammation cytokines or bacterial measures. All the tests were two-sided. A $P<0.05$ was accepted as the cutoff for statistical significance.

\section{RESULTS}

\section{Inpatients Characteristics}

During the time of study, a total of 528 adult inpatients with ScZ demonstrated a history of aggression behaviors prior to hospitalization. By excluding cases with $<2$ weeks of antipsychotics discontinuation (56 cases), aggression occurred prior to 1 week time period preceding hospital admission (135 cases), aggression occurred prior to and within 1 week (184 
TABLE 1 | Clinic characteristics of all inpatients at baseline.

\begin{tabular}{|c|c|c|c|c|}
\hline Items & $\begin{array}{l}\text { ScZ-Ag } \\
\text { group }\end{array}$ & $\begin{array}{l}\text { NScZ-Ag } \\
\text { group }\end{array}$ & Ctrl group & $P$-value \\
\hline Case No. & 112 & 112 & 56 & - \\
\hline Female, n (\%) & $67(59.8)$ & $64(57.1)$ & $35(62.5)$ & 0.792 \\
\hline Age, mean (SD), years & $33.5(8.4)$ & $34.2(9.1)$ & $33.8(8.7)$ & 0.835 \\
\hline BMl, mean (SD), kg/m² & $21.9(2.4)$ & $21.7(2.1)$ & $22.3(1.9)$ & 0.243 \\
\hline Ethnic Han, n (\%) & $105(93.8)$ & $109(97.3)$ & 52 (92.9) & 0.336 \\
\hline Low income, n (\%) & $72(64.3)$ & $41(36.7)$ & $17(30.4)$ & 0.000 \\
\hline Living with families, n (\%) & 91 (81.3) & $96(85.7)$ & $45(80.4)$ & 0.579 \\
\hline \multicolumn{5}{|l|}{ Marriage status, n (\%) } \\
\hline Married & $18(16.1)$ & $33(29.5)$ & $35(62.5)$ & 0.000 \\
\hline Single & $66(58.9)$ & $35(31.2)$ & $15(26.8)$ & \\
\hline Divorced & $26(23.1)$ & 37 (33.0) & $6(10.7)$ & \\
\hline Widowed & $2(1.9)$ & $7(6.3)$ & $0(0)$ & \\
\hline \multicolumn{5}{|l|}{ Education background, n (\%) } \\
\hline Elementary school and below & $82(73.2)$ & $49(43.8)$ & $12(21.4)$ & 0.000 \\
\hline Middle and high school & $15(13.4)$ & $39(34.8)$ & $16(28.6)$ & \\
\hline College and above & $15(13.4)$ & $24(21.5)$ & $28(50.0)$ & \\
\hline \multicolumn{5}{|l|}{ Occupation, n (\%) } \\
\hline Physical labor & $21(18.8)$ & $22(19.7)$ & $18(32.1)$ & 0.000 \\
\hline Mental labor & $13(11.6)$ & $10(8.9)$ & $29(51.8)$ & \\
\hline Unemployment & 78 (69.6) & $80(71.4)$ & $9(16.1)$ & \\
\hline No. of ScZ episodes \& & $5.6(2.7)$ & $4.9(3.2)$ & NA & 0.319 \\
\hline ScZ course, mean (SD), years\& & $7.4(4.3)$ & $6.9(4.1)$ & NA & 0.431 \\
\hline Total MOAS score ${ }^{\&}$ & $16.4(8.2)$ & $1.6(0.9)$ & $0(0.0)$ & 0.000 \\
\hline Total PANSS score\& & $65.2(8.3)$ & $63.4(7.5)$ & $0(0.0)$ & 0.090 \\
\hline
\end{tabular}

ScZ-Ag, schizophrenia with aggression; NScZ-Ag, schizophrenia without any aggression; BMI, body mass index; MOAS, Modified Overt Aggression Scale; PANSS, positive and negative syndrome scale; NA, not applicable; \&, analysis using Student's t-test between ScZ-Ag and NScZ-Ag groups. The meaning of the bold values indicate $P<0.05$.

cases), and aggression occurred only within 1 week but met the aforementioned exclusion criteria (41 cases), only $21.2 \%$ $(112 / 528)$ of them [average total MOAS score, mean(SD), 16.4(8.2)] were included in ScZ-Ag group as defined previously. In this study, 112 age-, gender-, and BMI-matched NScZ$\mathrm{Ag}$ inpatients [average total MOAS score, mean(SD), 1.6(0.9)] and 56 healthy volunteers were included. As Table 1 showed, there was statistical significance in terms of income, marriage, education level, and occupation among the three groups $(P<$ 0.001 for all variables). Compared with NScZ-Ag group, more aggression inpatients were single (58.9 vs. $31.2 \%, P=0.010)$ and a much higher proportion of aggression cases had low income (64.3 vs. $36.7 \%, P=0.017$ ) and poor education background (73.2 vs. $43.8 \%, P=0.021)$. Between inpatients with ScZ with and without aggression, there was no statistical difference regarding ethnicity, living conditions, occupation distribution, episodes with ScZ, course with ScZ, and total PANSS score $(P>0.05$ for all the variables).

\section{Inflammation and Severity of Aggression}

As shown in Figure 1, the results of ANCOVA analysis displayed that there were statistically significant differences between ScZ$\mathrm{Ag}, \mathrm{NScZ}-\mathrm{Ag}$, and Ctrl groups in terms of CRP $(F=75.2, P<$
0.001), IL-6 $(F=102.00, P<0.001)$, IL-1 $\beta(F=37.90, P<0.001)$, TNF- $\alpha(F=450.00, P<0.001)$, IL-17 $(F=7.00, P=0.007)$, and TGF- $\beta$ ( $F=7.55, P=0.008)$. Further, post-hoc analysis using Bonferroni's multiple comparison test found that none of inflammatory markers differed significantly between NScZ-Ag and Ctrl groups (all $P>0.05$ ), while serum levels of CRP, IL-6, IL$1 \beta$, and TNF- $\alpha$ dramatically increased approximately two to five times on average in ScZ-Ag group in comparison with NScZ-Ag group (all $P<0.001$ ). On partial correlation analysis controlling potential confounders, serum levels of CRP $(r=0.309, P<$ $0.001)$, IL-6 $(r=0.526, P<0.001)$, IL-1 $\beta(r=0.552, P<0.001)$, and TNF- $\alpha(r=0.517, P<0.001)$ were all positively associated with total MOAS score in ScZ-Ag group (Figure 2). Altogether, these results indicate that systemic proinflammation response mainly occurs in inpatients with $\mathrm{ScZ}$ with aggression behaviors.

\section{BT Determination and Its Association With Inflammation}

To explore the source of proinflammation phenotype, BTrelated serum biomarkers in all the subjects were measured (Figure 3). Regarding biomarkers of "leaky gut" (Claudin-3 and I-FABP), bacterial components (LPS and BactDNA), and LPSresponse products ( $\mathrm{SCD} 14$ and EndoCAb), statistically significant differences between the three groups were observed (all $P<0.01$ ) from ANCOVA analysis results. Post-hoc analysis showed that only BactDNA titers $(11.79 \pm 6.97$ vs. $7.19 \pm 4.76$ copies $/ \mu l, P$ $<0.001)$ and sCD14 levels $\left(1.57 \pm 1.15\right.$ vs. $1.07 \pm 0.61 \times 10^{6}$ $\mathrm{pg} / \mathrm{ml}, P<0.05)$ were moderately increased in NScZ-Ag group than Ctrl group, while serum concentrations of Claudin-3 (58.47 \pm 13.52 vs. $39.27 \pm 9.61 \mathrm{ng} / \mathrm{ml}, P<0.001)$, I-FABP $(80.47 \pm$ 21.47 vs. $29.56 \pm 7.46 \mathrm{pg} / \mathrm{ml}, P<0.001)$, LPS (73.51 \pm 32.29 vs. $23.16 \pm 7.83 \mathrm{pg} / \mathrm{ml}, P<0.001)$, sCD14 (3.45 \pm 1.39 vs. $1.57 \pm$ $\left.1.15 \times 10^{6} \mathrm{pg} / \mathrm{ml}, P<0.001\right)$ were significantly higher, EndoCAb concentration $\left(2.18 \pm 0.13\right.$ vs. $2.23 \pm 0.11 \log _{10} \mathrm{MMU} / \mathrm{ml}, P$ $<0.01$ ) was remarkably lower in ScZ-Ag group than NScZ-Ag group. In ScZ-Ag group (Table 2), circulating concentration of LPS was further found to be positively correlated with CRP ( $P$ $<0.001)$, IL-1 $\beta(P=0.001)$ and TNF- $\alpha(P=0.006)$, sCD14 was positively associated with CRP $(P<0.001)$, IL-6 $(P=0.007)$, and TNF- $\alpha(P=0.040)$ after controlling potential confounders. These data not only indicate the presence of "leaky gut," but also imply the link that circulating LPS from BT, as well as LPS responded sCD14, might be the important cause synergistically leading to the higher levels of proinflammation mediators observed in inpatients with $\mathrm{ScZ}$ with any type of aggression behaviors.

\section{Correlation of BT With Symptoms Dimensions}

Partial correlation analyses in inpatients with aggression (Table 3) showed that total MOAS score was positively associated with protein levels of circulating LPS $(r=0.412, P=0.005)$ or sCD14 ( $r=0.267, P=0.035)$. Regarding the subscale of MOAS, only aggression against objects $(r=0.406, P=0.006)$ or toward others $(r=0.326, P=0.011)$ were found to be correlated positively with circulating LPS, and such associations with the circulating sCD14 were also detected. In addition, results showed 


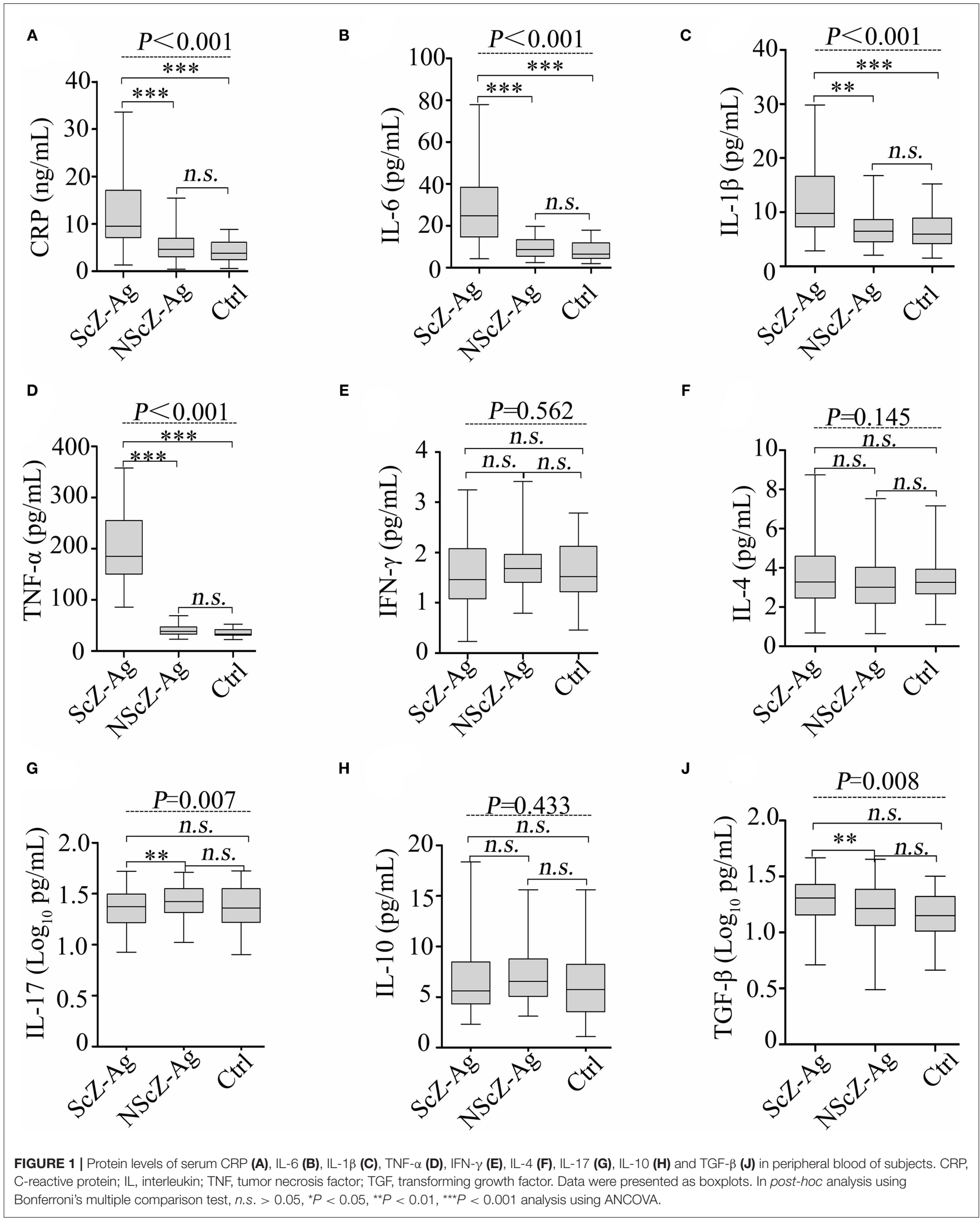


A

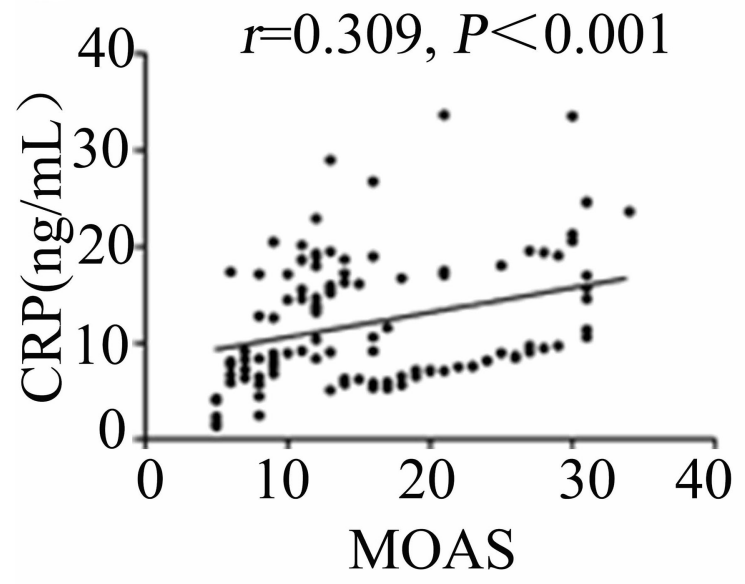

C

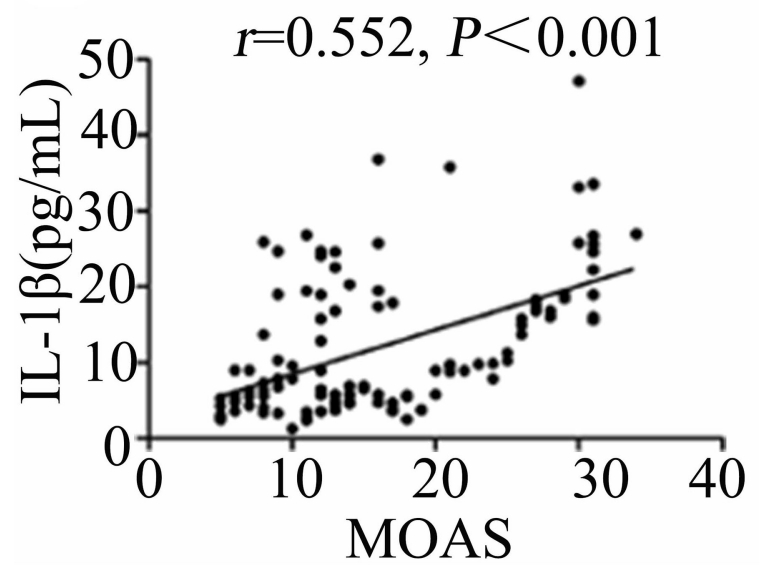

B

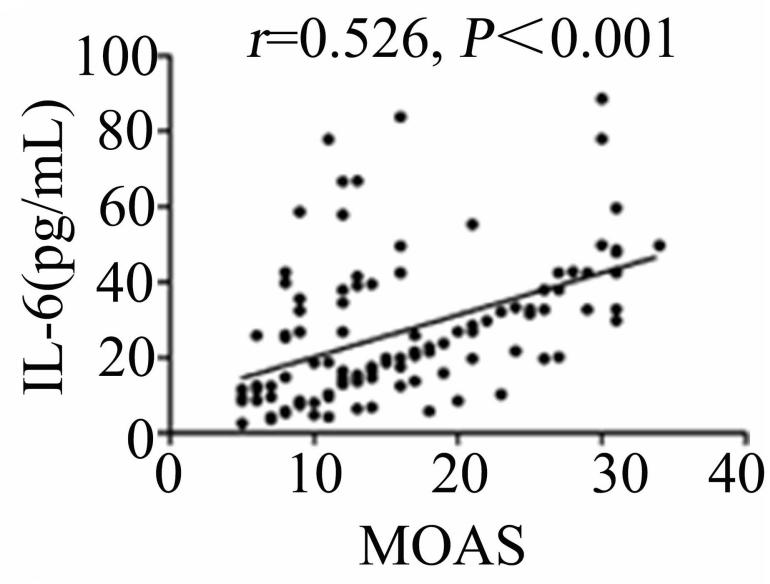

D

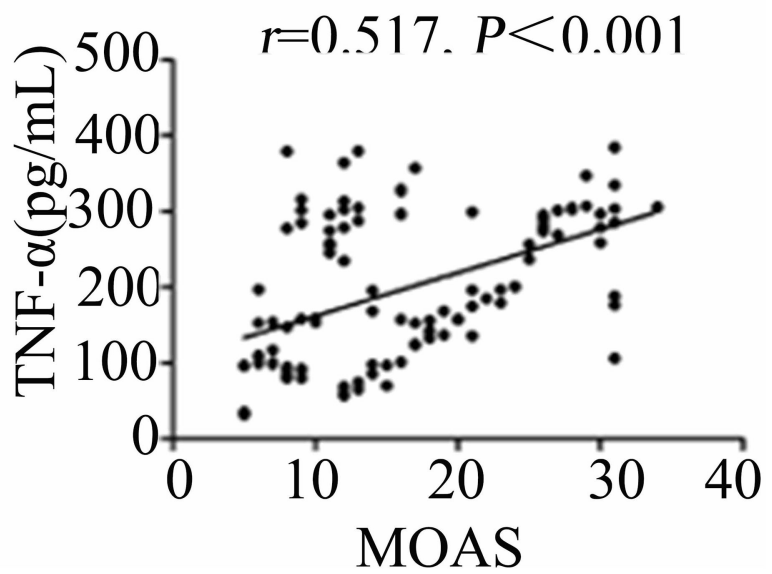

FIGURE 2 | Partial correlation analyses of MOAS with CRP (A), IL-6 (B), IL-1 $\beta$ (C), and TNF- $\alpha$ (D) in ScZ-Ag group.

statistically significant association between positive PANSS and circulating LPS $(r=0.298, P=0.023)$ or $\operatorname{sCD} 14(r=0.315$, $P=0.015)$. Altogether, the data further suggest that the increased protein levels of LPS or sCD14 in peripheral blood potentially initiate aggression behaviors in inpatients with ScZ via exacerbating the severity of systemic inflammation.

\section{DISCUSSION}

Aggression can attack individuals with or without psychosis. It is one of the top 20 causes of disabilities worldwide that is present in $15.3-53.2 \%$ of inpatients with ScZ in China (Zhou et al., 2016). Growing evidence demonstrate that the serious public health problem is the resultant of pro-/antiinflammation imbalance, since some inflammation cytokines were proved to be involved in the pathogenesis of ScZ (Müller et al., 2015; Petrikis et al., 2015; Momtazmanesh et al., 2019; Feng et al., 2020; Park and Miller, 2020). However, the role of and alterations in these cytokines may be variable in different stratifications of ScZ, antipsychotic drugs used or presence of aggression behaviors is a case (Petrikis et al., 2017; Momtazmanesh et al., 2019). This study was the first to focus on aggression-affected inpatients with ScZ with at least 2 weeks of antipsychotics discontinuation. Different from previous studies (Miller et al., 2011; de Witte et al., 2014; Momtazmanesh et al., 2019), our results from Bonferroni's multiple comparison tests demonstrated no difference in inflammation phenotype between inpatients with ScZ without aggression and healthy controls. The contradictory results may be attributed to differences in statistical analysis methods used and the specific enrolled participants without any aggression behaviors during the disease course, which further verifies the inconsistent conclusions regarding inflammatory phenotypes in ScZ (Momtazmanesh et al., 2019). In sharp contrast, dramatical elevations of CRP, IL-6, IL-1 $\beta$, and TNF- $\alpha$ were not only observed in inpatients with ScZ with aggression, but the elevated cytokines also correlated positively to the severity of aggression measured by MOAS score that is partly similar to the previous reports (Petrikis et al., 2015; Zhang et al., 


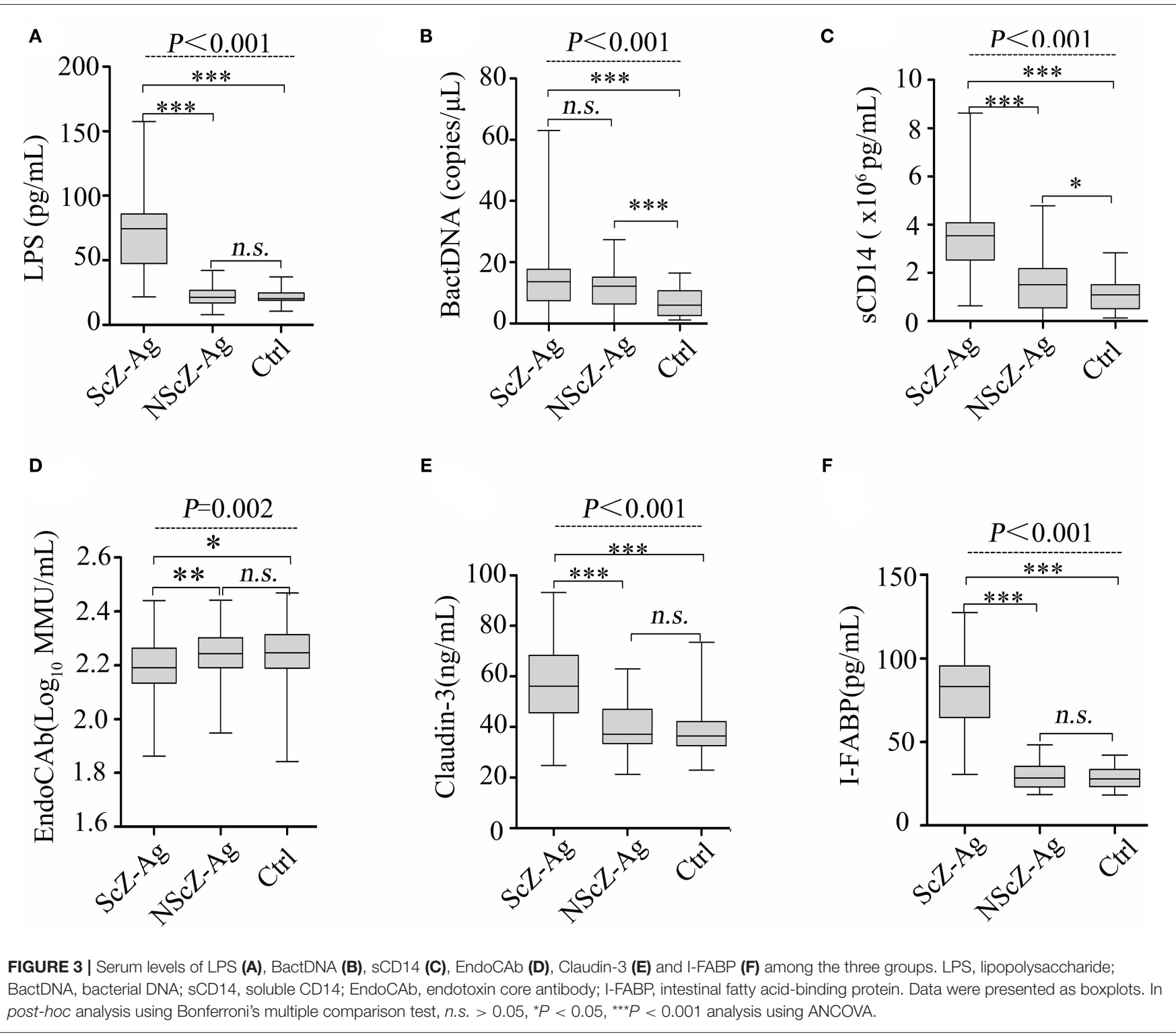

2017; Orsolini et al., 2018; Momtazmanesh et al., 2019; Fond et al., 2021). Li et al. demonstrated positive correlations between higher plasma IL-17 or TGF- $\beta 1$ and severity of aggression in patients with ScZ (Li et al., 2016), however, we found slightly lower serum IL-17 and higher serum TGF- $\beta 1$ in individuals with $\mathrm{ScZ}$ with aggression as compared with those without aggression. These findings indicate the need for additional research to confirm the role of IL-17 and TGF- $\beta 1$ in aggression onset. Among the functional redundancies of IL-6, IL-1 $\beta$, and TNF- $\alpha$, these proinflammation mediators potentially drive aggression in a sophisticated and coordinated network. These data collectively suggest the contributory role of systemic proinflammation in the occurrence of aggression in ScZ.

Gastrointestinal source of proinflammation was unveiled in deficit ScZ, and the leaky gut was identified as one of the prerequisites for the inflammatory pathophysiology (Severance et al., 2012, 2016; Barber et al., 2019; Ciháková et al., 2019; Maes et al., 2019b). Transcellular integrity, paracellular adherens, and tight junctions are demonstrated universally to be the structural basis for maintaining normal intestinal permeability. Permeability-related biomarkers such as I-FABP and Claudin3 present at high levels in peripheral blood can reliably reflect the occurrence of leaky gut as they are released into circulation by enterocytes when intestinal epitheliums are compromised (Barmeyer et al., 2017). The evidence that remarkable increases in serum levels of I-FABP and Claudin-3 only in cases with ScZ with aggression behaviors in this study indicates the possible role of increased intestinal permeability in the onset of proinflammation-driven aggression that has not been previously reported.

Correspondingly, the peripheral blood concentration of LPS was significantly higher in an aggression-affected group with 
TABLE 2 | Relations of bacterial translocation markers to cytokines in ScZ-Ag group.

\begin{tabular}{|c|c|c|c|c|c|c|c|c|}
\hline \multirow{2}{*}{ BT markers } & \multicolumn{2}{|c|}{ CRP } & \multicolumn{2}{|c|}{ IL-6 } & \multicolumn{2}{|c|}{ IL-1 $\beta$} & \multicolumn{2}{|c|}{ TNF- $\alpha$} \\
\hline & $r$ & $P$ & $r$ & $P$ & $r$ & $P$ & $r$ & $P$ \\
\hline LPS & 0.713 & $0.000^{\star}$ & 0.212 & 0.298 & 0.627 & $0.001^{*}$ & 0.583 & $0.006^{*}$ \\
\hline BactDNA & 0.201 & 0.329 & 0.196 & 0.472 & 0.098 & 0.592 & 0.302 & 0.129 \\
\hline sCD14 & 0.826 & $0.000^{*}$ & 0.509 & $0.007^{\star}$ & 0.056 & 0.613 & 0.341 & $0.040^{*}$ \\
\hline EndoCAb & -0.239 & 0.269 & -0.117 & 0.523 & -0.049 & 0.617 & -0.316 & 0.084 \\
\hline I-FABP & 0.112 & 0.564 & 0.082 & 0.613 & 0.067 & 0.627 & 0.298 & 0.158 \\
\hline Claudin-3 & 0.257 & 0.218 & 0.286 & 0.182 & 0.318 & 0.065 & 0.125 & 0.499 \\
\hline
\end{tabular}

${ }^{\star} P<0.05$. Analyses using partial correlation analysis. The meaning of the bold values indicate $P<0.05$.

TABLE 3 | Correlations of Lipopolysaccharide or SCD14 with severity aggression.

\begin{tabular}{|c|c|c|c|c|}
\hline \multirow[t]{2}{*}{ Items } & \multicolumn{2}{|c|}{ LPS } & \multicolumn{2}{|c|}{ sCD14 } \\
\hline & $r$ & $\boldsymbol{P}$ & $r$ & $\boldsymbol{P}$ \\
\hline Total MOAS score & 0.412 & $0.005^{\star}$ & 0.267 & $0.035^{*}$ \\
\hline Verbal aggression & 0.198 & 0.263 & 0.154 & 0.299 \\
\hline Aggression against objects & 0.406 & $0.006^{*}$ & 0.397 & $0.008^{*}$ \\
\hline Self-aggression & -0.054 & 0.512 & -0.067 & 0.476 \\
\hline Aggression toward others & 0.326 & $0.011^{*}$ & 0.256 & $0.042^{*}$ \\
\hline Total PANSS score & 0.068 & 0.477 & 0.118 & 0.364 \\
\hline Positive & 0.298 & $0.023^{\star}$ & 0.315 & $0.015^{\star}$ \\
\hline Negative & -0.136 & 0.317 & -0.098 & 0.418 \\
\hline General & 0.049 & 0.574 & 0.009 & 0.832 \\
\hline
\end{tabular}

${ }^{\star} P<0.05$. Analyses using partial correlation analysis. The meaning of the bold values indicate $P<0.05$.

ScZ as compared with the non-aggression. Translocating LPS links with an exacerbation of inflammation response (Panpetch et al., 2020) and the following correlation analysis also showed positive correlativity between the circulating concentrations of proinflammation mediators and LPS, and also LPS responded sCD14. Furthermore, serum levels of both the LPS and sCD14 were found to be related to specific aggression behaviors (aggression against objects or toward others) or psychotic symptoms (positive PANSS). As LPS-specific host response, sCD14 circulates at high levels in the serum and interacts with translocating LPS to stimulate antigen-presenting cells via toll-like receptor 4 (TLR4) signaling (Tsukamoto et al., 2018). Under bacteria or LPS challenge, vascular endothelial cells and perivascular mast cells have been reported to express abundant TLR4, thus, initiating the production of inflammation cytokines (Zeuke et al., 2002). On the other hand, decreased host EndoCAb in peripheral blood failed to bind and clear LPS from circulation, which ensures a high serum level of LPS for a long time and subsequently maintains systemic inflammation (Kyosiimire-Lugemwa et al., 2020). It is also worth noting that serum BactDNA loads in cases with ScZ with aggression may have little effect on inflammation state given the results from correlation analysis and differential expressions of BactDNA among cases with or without aggression. We can only speculate that serum BactDNA loads quantified by
qPCR likely underestimate the presence of BactDNA within whole blood and corresponding perturbation of inflammation markers may be transient. Collectively, these findings emphasize the implication of translocating LPS as well as sCD14 in the systemic inflammation response, and thus, argue for the potential causative relationship between BT and onset of aggression in ScZ.

Largely due to the failures of interpersonal inference, to develop a proper theory of mind or in sensory attenuation, ScZ was identified as one of the emotion recognition disorders (Demekas et al., 2020). Emotion recognition has also been suggested to underlie aggression in individuals with ScZ (Acland et al., 2021); however, that may be decreased by elevated lowgrade inflammation (Balter et al., 2018, 2021). In this study, evidence that systemic proinflammation potentially initiated by serum LPS correlated with aggression severity in inpatients with ScZ implies the possible contribution role of serum LPS to aggressive behaviors via emotion misrecognition that has important implications for integrated treatments of aggression.

Unfortunately, at least five limitations exist in our study. At first, a structured clinical interview to determine the clinical diagnosis of subjects was not performed. Second, only single samples from participants in a single center were obtained, within-subject verification of related biomarkers and replication procedures in larger study populations from multicenter are expected. Third, higher circulating BactDNA load was observed in NScZ-Ag group compared with the healthy group (Figure 3B), while correlation analysis between BactDNA and inflammation in NScZ-Ag group was not conducted as inflammation cytokines did not differ between the two groups (Figure 1), thereby perplexing the function of BactDNA in pathogenesis with ScZ. Furthermore, as with all case-controlled clinical studies, present data failed to adequately explain the causal relationship between BT-caused inflammation response and aggression in ScZ, related animal experiments are expected for ethical considerations. At last, the molecular mechanism by which translocating LPS promotes systemic inflammation and thus drives aggression remains to be further investigated.

\section{CONCLUSION}

In conclusion, this study verifies mainly the presence of leaky gut-caused BT and further correlates BT-derived LPS and 
soluble CD14 to the severity of aggression possibly by driving proinflammation response in cases with ScZ with aggression. These observations collectively indicate that BT may be a novel anti-inflammation therapeutic target for aggression prophylaxis and improving disease outcomes in patients with ScZ with aggression against objects and others.

\section{DATA AVAILABILITY STATEMENT}

The raw data supporting the conclusions of this article will be made available by the authors, without undue reservation.

\section{ETHICS STATEMENT}

The studies involving human participants were reviewed and approved by the Ethics Committee of the Second People's Hospital of Zhumadian. The patients/participants provided their written informed consent to participate in this study.

\section{REFERENCES}

Acland, E. L., Jambon, M., and Malti, T. (2021). Children's emotion recognition and aggression: a multi-cohort longitudinal study. Aggress. Behav. doi: 10.1002/ab.21989. [Epub ahead of print].

Balter, L., Hulsken, S., Aldred, S., Drayson, M. T., Higgs, S., Veldhuijzen van Zanten, J., et al. (2018). Low-grade inflammation decreases emotion recognition-evidence from the vaccination model of inflammation. Brain Behav. Immun. 73, 216-221. doi: 10.1016/j.bbi.2018. 05.006

Balter, L. J., Raymond, J. E., Aldred, S., Higgs, S., and Bosch, J. A. (2021). Age, BMI, and inflammation: associations with emotion recognition. Physiol. Behav. 232:113324. doi: 10.1016/j.physbeh.2021.113324

Barber, G. S., Sturgeon, C., Fasano, A., Cascella, N. G., Eaton, W. W., et al. (2019). Elevated zonulin, a measure of tight-junction permeability, may be implicated in schizophrenia. Schizophr. Res. 211, 111-112. doi: 10.1016/j.schres.2019.07.006

Barmeyer, C., Fromm, M., and Schulzke, J. D. (2017). Active and passive involvement of claudins in the pathophysiology of intestinal inflammatory diseases. Pflugers Arch. 469,15-26. doi: 10.1007/s00424-016-1914-6

Barzilay, R., Lobel, T., Krivoy, A., Shlosberg, D., Weizman, A., and Katz, N. (2016). Elevated C-reactive protein levels in schizophrenia inpatients is associated with aggressive behavior. Eur. Psychiatry 31, 8-12. doi: 10.1016/j.eurpsy.2015.09.461

Caso, J. R., Balanzá-Martínez, V., Palomo, T., and García-Bueno, B. (2016). The microbiota and gut-brain axis: contributions to the immunopathogenesis of schizophrenia. Curr. Pharm. Des. 22, 6122-6133. doi: $10.2174 / 1381612822666160906160911$

Charlson, F. J., Ferrari, A. J., Santomauro, D. F., Diminic, S., Stockings, E., Scott, J. G., et al. (2018). Global epidemiology and burden of schizophrenia: findings from the global burden of disease study 2016. Schizophr. Bull. 44, 1195-1203. doi: 10.1093/schbul/sby058

Chen, X., Xu, J., Wang, H., Luo, J., Wang, Z., Chen, G., et al. (2021). Profiling the differences of gut microbial structure between schizophrenia patients with and without violent behaviors based on $16 \mathrm{~S}$ rRNA gene sequencing. Int. J. Legal Med. 135, 131-141. doi: 10.1007/s00414-020-02439-1

Cho, W., Shin, W. S., An, I., Bang, M., Cho, D. Y., and Lee, S. H. (2019). Biological aspects of aggression and violence in schizophrenia. Clin. Psychopharmacol. Neurosci. 17, 475-486. doi: 10.9758/cpn.2019.17.4.475

Ciháková, D., Eaton, W. W., Talor, M. V., Harkus, U. H., and Demyanovich, H., Rodriguez, K., et al. (2019). Gut permeability and mimicry of the Glutamate Ionotropic Receptor NMDA type Subunit Associated with protein 1 (GRINA) as potential mechanisms related to a subgroup of people with schizophrenia

\section{AUTHOR CONTRIBUTIONS}

CW and TZ: contributed equally to the manuscript. J-YF and B-TC: methodology, supervision, visualization, and writingreview and editing. $\mathrm{CW}$ and $\mathrm{H}-\mathrm{XD}$ : clinical assessment, data curation, investigation, and writing-original draft. LH and X-LX: formal analysis and statistical analysis. All authors have contributed to and have approved the final manuscript.

\section{FUNDING}

This work was funded by the grant from the National Natural Science Foundation of China (82003337) and China Postdoctoral Science Foundation (2020M683268).

\section{ACKNOWLEDGMENTS}

We thank Prof Bing Cao (School of Public Health, Peking University) for her help in statistics and language editing.

with elevated antigliadin antibodies (AGA IgG). Schizophr. Res. 208, 414-419. doi: 10.1016/j.schres.2019.01.007

Das, S., Deuri, S. K., Sarmah, A., Pathak, K., Baruah, A., Sengupta, S., et al. (2016). Aggression as an independent entity even in psychosis-the role of inflammatory cytokines. J. Neuroimmunol. 292, 45-51. doi: 10.1016/j.jneuroim.2016.01.012

de Witte, L., Tomasik, J., Schwarz, E., Guest, P. C., Rahmoune, H., Kahn, R. S., et al. (2014). Cytokine alterations in first-episode schizophrenia patients before and after antipsychotic treatment. Schizophr. Res. 154, 23-29. doi: 10.1016/j.schres.2014.02.005

Demekas, D., Parr, T., and Friston, K. J. (2020). An investigation of the free energy principle for emotion recognition. Front. Comput. Neurosci. 14:30. doi: 10.3389/fncom.2020.00030

Ericsen, A. J., Lauck, M., Mohns, M. S., DiNapoli, S. R., Mutschler, J. P., Greene, J. M., et al. (2016). Microbial translocation and inflammation occur in hyperacute immunodeficiency virus infection and compromise host control of virus replication. PLoS Pathog. 12:e1006048. doi: 10.1371/journal.ppat.1006048

Feng, T., McEvoy, J. P., and Miller, B. J. (2020). Longitudinal study of inflammatory markers and psychopathology in schizophrenia. Schizophr. Res. 224, 58-66. doi: 10.1016/j.schres.2020.10.003

Fond, G., Sunhary de, Verville, P. L., Richieri, R., Etchecopar-Etchart, D., Korchia, T., et al. (2021). Redefining peripheral inflammation signature in schizophrenia based on the real-world FACE-SZ cohort. Prog. Neuropsychopharmacol. Biol. Psychiatry 111:110335. doi: 10.1016/j.pnpbp.2021.110335

Francés, R., Benlloch, S., Zapater, P., González, J. M., Lozano, B., Muñoz, C., et al. (2004). A sequential study of serum bacterial DNA in patients with advanced cirrhosis and ascites. Hepatology 39, 484-491. doi: 10.1002/hep.20055

Francés, R., González-Navajas, J. M., Zapater, P., Muñoz, C., Caño, R., Pascual, S., et al. (2007). Translocation of bacterial DNA from Gram-positive microorganisms is associated with a species-specific inflammatory response in serum and ascitic fluid of patients with cirrhosis. Clin. Exp. Immunol. 150, 230-237. doi: 10.1111/j.1365-2249.2007.03494.x

Huang, H. C., Wang, Y. T., Chen, K. C., Yeh, T. L., Lee, I. H., Chen, P. S., et al. (2009). The reliability and validity of the Chinese version of the modified overt aggression scale. Int. J. Psychiatry Clin. Pract. 13, 303-306. doi: 10.3109/13651500903056533

Kahn, R. S., Sommer, I. E., Murray, R. M., Meyer-Lindenberg, A., Weinberger, D. R., Cannon, T. D., et al. (2015). Schizophrenia. Nat. Rev. Dis. Primers 1:15067. doi: 10.1038/nrdp.2015.67

Kelley, M. E., White, L., Compton, M. T., and Harvey, P. D. (2013). Subscale structure for the Positive and Negative Syndrome Scale (PANSS): a proposed solution focused on clinical validity. Psychiatry Res. 205, 137-142. doi: 10.1016/j.psychres.2012.08.019 
Kyosiimire-Lugemwa, J., Anywaine, Z., Abaasa, A., Levin, J., Gombe, B., Musinguzi, K., et al. (2020). Effect of stopping cotrimoxazole preventive therapy on microbial translocation and inflammatory markers among human immunodeficiency virus-infected ugandan adults on antiretroviral therapy: the COSTOP trial immunology substudy. J. Infect. Dis. 222, 381-390. doi: 10.1093/infdis/jiz494

Lesh, T. A., Careaga, M., Rose, D. R., McAllister, A. K., Van de Water, J., Carter, C. S., et al. (2018). Cytokine alterations in first-episode schizophrenia and bipolar disorder: relationships to brain structure and symptoms. J. Neuroinflammation 15:165. doi: 10.1186/s12974-018-1197-2

Li, H., Zhang, Q., Li, N., Wang, F., Xiang, H., Zhang, Z., et al. (2016). Plasma levels of Th17-related cytokines and complement C3 correlated with aggressive behavior in patients with schizophrenia. Psychiatry Res. 246, 700-706. doi: 10.1016/j.psychres.2016.10.061

Maes, M., Sirivichayakul, S., Kanchanatawan, B., and Vodjani, A. (2019a). Breakdown of the paracellular tight and adherens junctions in the gut and blood brain barrier and damage to the vascular barrier in patients with deficit schizophrenia. Neurotox. Res. 36, 306-322. doi: 10.1007/s12640-019-00054-6

Maes, M., Sirivichayakul, S., Kanchanatawan, B., and Vodjani, A. (2019b). Upregulation of the intestinal paracellular pathway with breakdown of tight and adherens junctions in deficit schizophrenia. Mol. Neurobiol. 56, 7056-7073. doi: 10.1007/s12035-019-1578-2

Manchia, M., and Fanos, V. (2017). Targeting aggression in severe mental illness: the predictive role of genetic, epigenetic, and metabolomic markers. Progr. Neuro Psychopharmacol. Biol. Psychiatry 77, 32-41. doi: 10.1016/j.pnpbp.2017.03.024

Martin-Subero, M., Anderson, G., Kanchanatawan, B., Berk, M., and Maes, M. (2016). Comorbidity between depression and inflammatory bowel disease explained by immune-inflammatory, oxidative, and nitrosative stress; tryptophan catabolite; and gut-brain pathways. CNS Spectr. 21, 184-198. doi: $10.1017 /$ S1092852915000449

Miller, B. J., Buckley, P., Seabolt, W., Mellor, A., and Kirkpatrick, B. (2011). Meta-analysis of cytokine alterations in schizophrenia: clinical status and antipsychotic effects. Biol. Psychiatry 70, 663-671. doi: 10.1016/j.biopsych.2011.04.013

Misiak, B., Łoniewski, I., Marlicz, W., Frydecka, D., Szulc, A., Rudzki, L., et al. (2020). The HPA axis dysregulation in severe mental illness: can we shift the blame to gut microbiota? Progr. Neuro Psychopharmacol. Biol. Psychiatry 102:109951. doi: 10.1016/j.pnpbp.2020.109951

Momtazmanesh, S., Zare-Shahabadi, A., and Rezaei, N. (2019). Cytokine alterations in schizophrenia: an updated review. Front. Psychiatry 10:892. doi: 10.3389/fpsyt.2019.00892

Müller, N., Weidinger, E., Leitner, B., and Schwarz, M. J. (2015). The role of inflammation in schizophrenia. Front. Neurosci. 9:372. doi: $10.3389 /$ fnins.2015.00372

Orsolini, L., Sarchione, F., Vellante, F., Fornaro, M., Matarazzo, I., Martinotti, G., et al. (2018). Protein-C reactive as biomarker predictor of schizophrenia phases of illness? a systematic review. Curr. Neuropharmacol. 16, 583-606. doi: 10.2174/1570159X16666180119144538

Panpetch, W., Hiengrach, P., Nilgate, S., Tumwasorn, S., Somboonna, N., Wilantho, A., et al. (2020). Additional Candida albicans administration enhances the severity of dextran sulfate solution induced colitis mouse model through leaky gut-enhanced systemic inflammation and gut-dysbiosis but attenuated by Lactobacillus rhamnosus L34. Gut Microbes 11, 465-480. doi: $10.1080 / 19490976.2019 .1662712$

Park, S., and Miller, B. J. (2020). Meta-analysis of cytokine and Creactive protein levels in high-risk psychosis. Schizophr. Res. 226, 5-12. doi: 10.1016/j.schres.2019.03.012

Petra, A. I., Panagiotidou, S., Hatziagelaki, E., Stewart, J. M., Conti, P., and Theoharides, T. C. (2015). Gut-microbiota-brain axis and its effect on neuropsychiatric disorders with suspected immune dysregulation. Clin. Ther. 37, 984-995. doi: 10.1016/j.clinthera.2015.04.002

Petrikis, P., Voulgari, P. V., Tzallas, A. T., Archimandriti, D. T., Skapinakis, P., and Mavreas, V. (2015). Cytokine profile in drug-naïve, first episode patients with psychosis. J. Psychosom. Res. 79, 324-327. doi: 10.1016/j.jpsychores.2015.06.011
Petrikis, P., Voulgari, P. V., Tzallas, A. T., Boumba, V. A., Archimandriti, D. T., Zambetas, D., et al. (2017). Changes in the cytokine profile in first-episode, drug-naïve patients with psychosis after short-term antipsychotic treatment. Psychiatry Res. 256, 378-383. doi: 10.1016/j.psychres.2017.07.002

Severance, E. G., Alaedini, A., Yang, S., Halling, M., Gressitt, K. L., Stallings, C. R., et al. (2012). Gastrointestinal inflammation and associated immune activation in schizophrenia. Schizophr. Res. 138, 48-53. doi: 10.1016/j.schres.2012.02.025

Severance, E. G., Dickerson, F., and Yolken, R. H. (2020). Complex gastrointestinal and endocrine sources of inflammation in schizophrenia. Front. Psychiatry 11:549. doi: 10.3389/fpsyt.2020.00549

Severance, E. G., Yolken, R. H., and Eaton, W. W. (2016). Autoimmune diseases, gastrointestinal disorders and the microbiome in schizophrenia: more than a gut feeling. Schizophr. Res. 176, 23-35. doi: 10.1016/j.schres.2014.06.027

Singh, L., Kaur, A., Bhatti, M. S., and Bhatti, R. (2019). Possible molecular mediators involved and mechanistic insight into fibromyalgia and associated co-morbidities. Neurochem. Res. 44, 1517-1532. doi: 10.1007/s11064-019-02805-5

Stepnicki, P., Kondej, M., and Kaczor, A. A. (2018). Current concepts and treatments of schizophrenia. Molecules 23:2087. doi: $10.3390 /$ molecules 23082087

Such, J., Francés, R., Muñoz, C., Zapater, P., Casellas, J. A., and Cifuentes, A. (2002). Detection and identification of bacterial DNA in patients with cirrhosis and culture-negative, nonneutrocytic ascites. Hepatology 36, 135-141. doi: $10.1053 /$ /hep.2002.33715

Tiihonen, J., Mittendorfer-Rutz, E., Majak, M., Mehtälä, J., Hoti, F., Jedenius, E., et al. (2017). Real-world effectiveness of antipsychotic treatments in a nationwide cohort of 29823 patients with schizophrenia. JAMA Psychiatry 74, 686-693. doi: 10.1001/jamapsychiatry.2017.1322

Tsukamoto, H., Takeuchi, S., Kubota, K., Kobayashi, Y., Kozakai, S., Ukai, I., et al. (2018). Lipopolysaccharide (LPS)-binding protein stimulates CD14-dependent Toll-like receptor 4 internalization and LPS-induced TBK1-IKK $\epsilon$-IRF3 axis activation. J. Biol. Chem. 293, 10186-10201. doi: 10.1074/jbc.M117.796631

Zeng, C., Yang, P., Cao, T., Gu, Y., Li, N., Zhang, B., et al. (2021). Gut microbiota: An intermediary between metabolic syndrome and cognitive deficits in schizophrenia. Progr. Neuro Psychopharmacol. Biol. Psychiatry 106:110097. doi: 10.1016/j.pnpbp.2020.110097

Zeuke, S., Ulmer, A. J., Kusumoto, S., Katus, H. A., and Heine, H. (2002). TLR4mediated inflammatory activation of human coronary artery endothelial cells by LPS. Cardiovasc. Res. 56, 126-134. doi: 10.1016/S0008-6363(02)00512-6

Zhang, Q., Hong, W., Li, H., Peng, F., Wang, F., Li, N., et al. (2017). Increased ratio of high sensitivity C-reactive protein to interleukin-10 as a potential peripheral biomarker of schizophrenia and aggression. Int. J. Psychophysiol. 114, 9-15. doi: 10.1016/j.ijpsycho.2017.02.001

Zhou, J. S., Zhong, B. L., Xiang, Y. T., Chen, Q., Cao, X. L., Correll, C. U., et al. (2016). Prevalence of aggression in hospitalized patients with schizophrenia in China: a meta-analysis. Asia Pac. Psychiatry 8, 60-69. doi: 10.1111/appy.12209

Conflict of Interest: The authors declare that the research was conducted in the absence of any commercial or financial relationships that could be construed as a potential conflict of interest.

Publisher's Note: All claims expressed in this article are solely those of the authors and do not necessarily represent those of their affiliated organizations, or those of the publisher, the editors and the reviewers. Any product that may be evaluated in this article, or claim that may be made by its manufacturer, is not guaranteed or endorsed by the publisher.

Copyright $\odot 2021$ Wang, Zhang, He, Fu, Deng, Xue and Chen. This is an open-access article distributed under the terms of the Creative Commons Attribution License (CC $B Y)$. The use, distribution or reproduction in other forums is permitted, provided the original author(s) and the copyright owner(s) are credited and that the original publication in this journal is cited, in accordance with accepted academic practice. No use, distribution or reproduction is permitted which does not comply with these terms. 